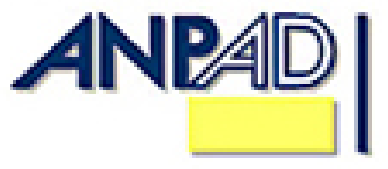

Disponível em

http://www.anpad.org.br/rac

RAC, Rio de Janeiro, v. 16, n. 1, art. 7, pp. 118-136, Jan./Fev. 2012

$(\mathrm{cc})$ EY-No

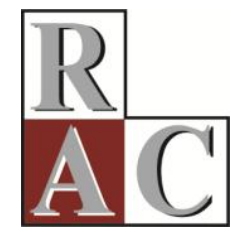

\title{
Orientação para Aprendizagem, Orientação para Mercado e Desempenho Organizacional: Evidências Empíricas
}

\section{Learning Orientation, Market Orientation and Organizational Performance: Empirical Evidences}

\author{
Eduardo Botti Abbade * \\ E-mail: eduardo@unifra.br \\ Universidade Federal do Rio Grande do Sul - UFRGS \\ Porto Alegre, RS, Brasil. \\ Roselaine Ruviaro Zanini \\ E-mail: rrzanini@terra.com.br \\ Universidade Federal de Santa Maria - UFSM \\ Santa Maria, RS, Brasil. \\ Adriano Mendonça Souza \\ E-mail: amsouza@ccne.ufsm.br \\ Universidade Federal de Santa Maria - UFSM \\ Santa Maria, RS, Brasil.
}

\footnotetext{
* Endereço: Eduardo Botti Abbade

Rua Riveira, 202, apto. 402, Petrópolis, Porto Alegre/RS, 90670-160.
}

Copyright (C) 2012 RAC. Todos os direitos, até mesmo de tradução, são reservados. É permitido citar parte de artigos sem autorização prévia, desde que seja identificada a fonte. 


\title{
Resumo
}

Este estudo tem como objetivo identificar de que forma a Orientação para Aprendizagem (OPA) e a Orientação para Mercado (OPM) influenciam o desempenho das empresas da região central do Rio Grande do Sul. O método empregado foi uma pesquisa de levantamento com 123 empresas da região central do RS. O instrumento de coleta de dados foi elaborado utilizando a escala de OPA (Sinkula, Baker, \& Noordewier, 1997), a escala MARKOR (Kohli, Jaworski, \& Kumar, 1993) e itens de avaliação do desempenho organizacional, propostos por Narver e Slater (1990) e Baker e Sinkula (1999). Os resultados sugerem que a OPM exerce influência positiva e significativa no desempenho organizacional das empresas pesquisadas. Foi constatado também que a OPM influencia significativamente o Desempenho Organizacional, quando intermediada pela OPA; assim como a OPA exerce influência significativa no desempenho das organizações, quando intermediada pela OPM.

Palavras-chaves: orientação para mercado; orientação para aprendizagem; estratégia; desempenho organizacional; equações estruturais.

\begin{abstract}
This study aims to identify how learning orientation (LO) and market orientation (MO) influence the performance of enterprises in the central region of the state of Rio Grande do Sul (RS), Brazil. The method used involved a survey of 123 companies in central RS. The instrument for data collection was developed using the LO scale (Sinkula, Baker, \& Noordewier, 1997), the MARKOR scale (Kohli, Jaworski, \& Kumar, 1993) and items for evaluation of organizational performance proposed by Narver and Slater (1990) and Baker and Sinkula (1999). The results suggest that MO has a significant positive influence on the organizational performance of the companies surveyed. It was also noted that the MO significantly influences organizational performance when mediated by the LO, just as the LO has a significant influence on organizational performance when mediated by MO.
\end{abstract}

Key words: market orientation; learning orientation; strategy; organizational performance; structural equations. 


\section{Introdução}

Atualmente as organizações, em sua busca incessante por vantagens competitivas que possam proporcionar melhores desempenhos organizacionais, adotam posturas orientadas para fins específicos. Múltiplas estratégias e ações organizacionais são encontradas, proporcionando resultados diversos nos mais variados tipos e estruturas organizacionais. Dentre as orientações e posturas organizacionais encontra-se o conceito de organização orientada para o mercado.

A orientação para mercado (OPM) passou a ter evidência na administração e entre os acadêmicos de marketing, devido às alterações sociais que mudaram o relacionamento das organizações e outras entidades com os seus clientes e públicos de interesse. Estudos sobre a OPM aumentaram significativamente a partir de 2001, sendo grande parte deles desenvolvidos no âmbito europeu e americano (Liao, Chang, Wu, \& Katrichis, 2011).

A organização orientada para o mercado tem uma capacidade superior de relacionamento com o mercado, a qual consiste em maior lealdade dos clientes e, consequentemente, em vantagens sustentáveis, fundamentadas na reciprocidade, compromissos mútuos, confiança e elos, passando pelo compartilhamento de informações, parceria cooperativa e coordenação conjunta de processos para o desenvolvimento de tecnologia e produtos. $\mathrm{O}$ aumento da lealdade leva à maior lucratividade, primeiramente, por ajudar a manter a base de clientes, na medida em que sua perda fica mais lenta e, por consequência, clientes leais são mais lucrativos; como os custos para o seu atendimento são menores, as compras tendem a ser maiores, ocorrendo uma diminuição da sensibilidade em relação aos preços, bem como uma divulgação boca a boca favorável (Day, 2001).

Outra postura de orientação e direcionamento organizacional que tem chamado atenção, tanto do meio acadêmico como do meio empresarial, é a Orientação para Aprendizagem (OPA). Fundamentada nas teorias e perspectivas da Gestão do Conhecimento e da Aprendizagem Organizacional, esta postura estratégica é vinculada diretamente à cultura da organização, sendo considerada mais uma filosofia organizacional do que postura estratégica propriamente dita.

A base da organização que aprende, foco da organização orientada para a aprendizagem, está na estrutura organizacional, que deve facilitar a geração, a transferência e a ampliação do conhecimento. Enquanto a OPM é considerada característica organizacional, que direciona e prioriza as atividades de coleta, disseminação e utilização das informações do mercado, a OPA é característica organizacional que afeta a propensão de uma organização em gerar valor e aprender por meio de mecanismos de circuito duplo (double-loop learning). A OPM é determinada pela produção, disseminação e adoção do conhecimento. A OPA focaliza suas ações e planejamentos em posturas questionadoras, onde o conhecimento existente é constantemente avaliado e questionado (Sinkula, Baker, \& Noordewier, 1997), propondo assim formas de inovar e melhorar as posturas e ações estratégicas organizacionais.

Para obter melhor desempenho organizacional é necessário que as empresas, assim como seus gestores, planejem e implementem ações que direcionem a empresa para uma postura Orientada para o Mercado e Orientada para a Aprendizagem. Acredita-se que estas orientações incrementam o desempenho organizacional, assim como também se complementam entre si. Estudos que buscam desenvolver essa temática e que relacionam a aprendizagem com o desempenho organizacional não são novidade no meio acadêmico, tanto em nível internacional (Baker \& Sinkula, 1999; Cantalone, Cavusgil, \& Zhao, 2002; Rhee, Park, \& Lee, 2010; Wang, 2008), como no âmbito nacional (Leopoldino \& Loiola, 2010; Perin, Sampaio, \& Faleiro, 2004). Também são encontrados estudos importantes que relacionam a OPM e o Desempenho Organizacional (Baker \& Sinkula, 1999; Ellis, 2006; Jiménez \& Navarro, 2007; Perin \& Sampaio, 2001; Olavarrieta \& Friedmann, 2008).

Entretanto cabe investigar qual o nível de impacto que essas orientações têm no desempenho organizacional, assim como o impacto que cada uma apresenta na outra. Desta forma, o presente estudo tem como objetivo geral identificar de que forma a OPA e a OPM influenciam o desempenho das empresas da região central do Rio Grande do Sul. 
Este estudo se justifica por existir uma necessidade de maior aprofundamento teórico-empírico da relação existente entre OPA, a OPM e o desempenho organizacional. Com isso, busca-se contribuir, de modo empírico, com a construção de um modelo baseado em modelagem de equações estruturais a respeito das relações de interdependência existentes entre os constructos. Também se salienta que este estudo apresenta uma validade regional, já que as empresas estudadas são atuantes na região central do Rio Grande do Sul. Busca-se também contribuir para o campo prático com resultados empíricos, que poderão proporcionar melhor compreensão da relação existente entre a OPA, a OPM e o desempenho organizacional, gerando, assim, melhores condições para o processo de planejamento e tomada de decisão.

\section{A Orientação para Mercado}

Podendo ser considerado um tema de grande impacto no meio acadêmico e empresarial, a OPM tem adquirido um espaço de grande relevância nos estudos referentes às estratégias de marketing $\mathrm{e}$ desempenho organizacional. Grande parte de seu desenvolvimento é da década de 90, com contribuições de Kohli e Jaworski (1990), Kohli, Jaworski e Kumar (1993) e Narver e Slater (1990; 1995); assim, a OPM pode ser encarada como forma de as empresas se posicionarem no mercado de forma a obterem um melhoramento em seus resultados operacionais e estratégicos.

A OPM é definida como a cultura organizacional que desenvolve os comportamentos necessários à criação de valor elevado para o cliente, de forma mais eficaz e eficiente, gerando vantagem competitiva para a empresa (Narver \& Slater, 1990). Assim, com o foco central nos clientes e no mercado, a OPM é um conceito proveniente da estrutura conceitual do marketing. Considera-se a OPM mais adequada do que a Orientação para o Marketing, já que a primeira focaliza suas atenções no mercado e nos clientes (aspectos externos à empresa), além de focar nos aspectos do planejamento de marketing (Kohli \& Jaworski, 1990).

Uma empresa orientada para o mercado pode oferecer soluções que possam ir além das expectativas de valor dos seus clientes. Este tipo de empresa sabe que existem várias formas para reduzir os custos totais de aquisição e de uso para os compradores, assim como também existem muitas maneiras de criar benefícios adicionais; é por isso que todas as alternativas são avaliadas, para se identificar qual forma poderá ser mais efetiva na criação do valor superior e sustentável da empresa, para os clientes-alvo atuais e futuros. Além disso, é necessário ainda desenvolver uma visão de longo prazo nos relacionamentos, interligando a cultura e a estrutura organizacional efetivamente, coordenando os esforços na criação de um valor superior para o cliente, em todos os setores da empresa, não deixando isso ser apenas responsabilidade do marketing (Narver \& Slater, 1990).

Entretanto, para que uma empresa obtenha maior vantagem competitiva, é importante que ela esteja sempre atualizada (Narver \& Slater, 1995). Assim, considera-se a aprendizagem organizacional como forma de que a empresa aprenda mais rapidamente que seus concorrentes. A dedicação a programas de treinamento e as mudanças organizacionais frequentes, contudo, podem provocar uma falha no desenvolvimento de uma cultura organizacional orientada para o mercado (Narver, Slater, \& Tietje, 1998).

Após o reconhecimento da importância da OPM, e aceitar a existência de lacunas em seu desenvolvimento teórico, Kohli e Javorski (1990, p. 1) defenderam a necessidade de "prover uma definição operacional, desenvolver um levantamento de proposições e construir um arcabouço compreensivo para orientar futuras pesquisas". Assim, os autores partiram para a definição do construto, fundamentando-o na literatura de marketing, entre outras disciplinas, e em pesquisa de campo, realizadas com executivos colocados nas mais diversas funções e níveis hierárquicos das organizações. Por meio do afrontamento das visões dos executivos e da literatura, Kohli e Jaworski (1990) localizaram evidências que os levaram a indicar que a OPM consiste em três aspectos fundamentais: (a) geração de inteligência; (b) disseminação de inteligência; e (c) responsividade. 
Conforme os autores: “orientação para mercado é a ampla geração de inteligência de mercado concernente às necessidades dos atuais e futuros clientes, disseminação da inteligência através dos departamentos e a ampla resposta a elas" (Kohli \& Jaworski, 1990, p. 6).

Com relação à geração de inteligência, é possível constatar que o ponto de partida da OPM é a inteligência do mercado, a qual é definida com conceito que envolve uma análise dos fatores externos que afetam o mercado, além do conhecimento das necessidades e preferências dos clientes, como pode ser visto nas regulamentações governamentais, na tecnologia, entre outros (Kohli \& Jaworski, 1990). Dessa maneira, a geração de inteligência compreende pesquisas com consumidores e mecanismos complementares, tais como reuniões com clientes, discussões com parceiros e análise de relatórios de vendas. A tentativa de envolver a análise dos fatores externos na geração de inteligência está ligada à necessidade de conhecer as ações dos competidores e a maneira como elas influenciam as preferências dos clientes. Outro fator que influencia a geração de inteligência é o ambiente de negócios (Jaworski, Macinnis, \& Kohli, 2002). Assim, considera-se a necessidade de reconhecer as pressões do mercado e os sistemas ambientais, entre outros aspectos, como sendo uma etapa do processo de geração de inteligência competitiva. Para a disseminação da inteligência, essa prática deve ser realizada por meio de comunicação horizontal, a qual deve ocorrer dentro da empresa, envolvendo todos os departamentos (Kohli \& Jaworski, 1990).

Já a responsividade configura-se nas ações adotadas para responder à geração e à disseminação da inteligência. Fundamentalmente, a responsividade é composta de dois conjuntos de atividades: (a) concepção de resposta; e (b) implementação de resposta. A primeira foca no uso da inteligência de mercado para desenvolver planos; e a segunda, na consecução de ditos planos (Kohli \& Jaworski, 1990). A responsividade envolve a seleção de mercados-alvo, o desenho e a oferta de produtos que respondam às necessidades atuais e futuras dos clientes, etc. (Kohli, Jaworski, \& Kumar, 1993).

O estudo de Kohli e Jaworski (1990) assim como o de Narver e Slater (1990) buscaram delimitar e estruturar o conceito de OPM, de modo a propor um constructo a ser validado e adotado como ferramenta de diagnóstico. Inicialmente os autores Narver e Slater (1990) propuseram um constructo unidimensional, composto por três perspectivas a serem analisadas: (a) a orientação para o cliente; (b) a orientação para os concorrentes; e (c) a coordenação interfuncional do negócio.

Já Kohli e Jaworski (1990), também estruturando o constructo proposto em três perspectivas a serem abordadas, propuseram que a OPM seria mensurada por meio da (a) identificação da geração de inteligência de mercado pela unidade de negócio; (b) a disseminação dessa inteligência de mercado; e (c) a resposta da unidade de negócio à inteligência gerada e disseminada anteriormente. A validade empírica desse modelo e suas proposições foram testadas pelos seus autores com o objetivo de elaborar e validar a formulação de um modelo de avaliação e mensuração de OPM de uma unidade de negócio, baseado na proposição dos antecedentes e consequências da OPM. Este modelo validado deu origem então à escala MARKOR (Kohli et al., 1993), que é a ferramenta de avaliação utilizada neste estudo.

A partir do delineamento do construto de OPM e de escalas para sua mensuração, a verificação empírica da relação entre OPM e desempenho tem sido foco central de diversos estudos, conduzidos em diferentes indústrias, setores e contextos culturais. De maneira geral, é observada uma relação positiva entre OPM e desempenho empresarial (Perin, 2002; Sampaio, 2000).

Ao definir a OPM, Kohli et al. (1993) estabeleceram uma cadeia em que consideram as condições que antecedem a OPM, as consequências da orientação e os fatores moderadores. Para os autores, como consequências da OPM, ficam fortalecidos o comprometimento organizacional e o espírito de equipe, que é gerado nos empregados. Os achados de Kohli e Jaworski (1990) revelaram que as respostas dos executivos entrevistados direcionam para o benefício tanto social quanto psicológico dos empregados envolvidos em uma situação de OPM. Ainda, os autores relataram as considerações dos executivos sobre o sentimento de pertencer à organização, quando todos trabalham pelo objetivo comum de satisfazer os clientes. 
A outra consequência da OPM é a melhoria do desempenho organizacional, segundo as pesquisas de Kohli et al. (1993). Em geral, as variáveis de desempenho utilizadas são o ROI (Return Over Investments), o volume de vendas, e a participação no mercado. No entanto, a intensidade da relação entre a OPM e o desempenho pode variar de acordo com o perfil das empresas.

Estudos brasileiros e em setores específicos investigaram a relação entre a OPM e o desempenho organizacional como no setor metal mecânico (Mandelli, 1999), confecções (Menna, 2001), setor calçadista gaúcho (Silveira, 1998) e no setor de ensino superior (Antoni, 2004). Outros estudos internacionais também investigaram a OPM, apresentando resultados interessantes. Ellis (2006) observou que o valor da OPM enfraquece na proporção do distanciamento cultural que separa o mercado doméstico dos EUA. Outro estudo importante sugere, a partir de seus resultados, que a influência da OPM na performance organizacional só é significativa quando mediada pela OPA (Jiménez \& Navarro, 2007).

Já outra pesquisa acadêmica interessante apresenta resultados de impacto significativo da OPM, do senso de mercado e da inovatividade no desempenho superior das organizações (Olavarrieta \& Friedmann, 2008). E, tendo como foco empresas de pequeno e médio porte, Armario, Ruiz e Armario (2008) investigam a relação entre a OPM, a aquisição de conhecimento, o comprometimento com o mercado e suas inter-relações na internacionalização das empresas, atestando relações significativas entre os constructos.

\section{Gestão do Conhecimento e Orientação para Aprendizagem}

A gestão do conhecimento e a aprendizagem organizacional são encaradas como estratégias para as empresas se manterem atualizadas, em face das turbulências e exigências do mercado competitivo, elevando, assim, o desempenho organizacional (Khandekar \& Sharma, 2006; Prieto \& Revilla, 2006). O conhecimento é visto como fonte de vantagem competitiva para as empresas que visam ao seu desenvolvimento. Gerar e transferir o conhecimento nas empresas são processos de aprendizagem organizacional, considerados de grande importância estratégica para o desenvolvimento competitivo. Além disso, mais importante do que gerar novos conhecimentos é fazer conexões com conhecimentos já existentes, ampliando a rede de relacionamentos internos e externos da organização (Eboli, 2004).

Independentemente da estratégia adotada, atualmente o gerenciamento efetivo do conhecimento corporativo é capaz de garantir às empresas o diferencial competitivo necessário para levá-las a uma posição de liderança. Surge então a gestão estratégica do conhecimento corporativo, definido como a tarefa de identificar, desenvolver, disseminar e atualizar o conteúdo estrategicamente relevante para a empresa, por meio de processos internos ou externos (Fleury \& Oliveira, 2001).

Simon (1991) afirma que a aprendizagem organizacional ocorre de duas maneiras distintas: (a) quando os membros da organização aprendem; e (b) quando ocorre a contratação de pessoas que tragam novos conhecimentos à organização. Com isso, considera-se que o conhecimento se encontra depositado principalmente nas pessoas, as quais são os elementos da aprendizagem e que constroem novos conhecimentos e geram a aprendizagem por meio de processos experienciais (Kolb, 1984) e cognitivos (Senge, 1990). Assim, partindo desse pressuposto, é possível inferir que as características individuais e psicológicas podem vir a influenciar o modo como as pessoas aprendem e disseminam novos conhecimentos.

Entretanto, como pressuposto básico para a gestão da aprendizagem e do conhecimento organizacional reside no fato de que é necessário criar, adquirir e transferir conhecimentos (Garvin, 1993) que permitam que a organização modifique suas rotinas, estratégias e redirecione seu foco de mercado. Objetiva-se com isso evitar que a organização fique excessivamente vinculada ao conhecimento depositado em seus funcionários, disseminando o conhecimento no ambiente 
organizacional. Como forma de pensar a aprendizagem organizacional, encontra-se o trabalho seminal de Argyris e Schön (1978) e o modelo da aprendizagem em circuito simples e duplo. Para os autores, quando um erro ou desvio é detectado e corrigido, sem questionar ou alterar os valores subjacentes ao sistema, ocorre uma aprendizagem em circuito simples. Desse modo, apenas a ação foi alterada sem ser realizado nenhum questionamento a respeito dos valores e teorias postas em prática. Já a aprendizagem em circuito duplo representa a forma de aprender por meio do questionamento dos valores e teorias em uso. A aprendizagem em circuito simples é indicada para rotinas e aspectos repetitivos da organização. Em contrapartida, a aprendizagem em circuito duplo é mais indicada para aspectos complexos e não programáveis da organização, já que esta busca mudanças mais profundas e consideradas estratégicas para uma organização.

Narver e Slater (1995, p. 67) propõem um modelo em que a aprendizagem organizacional é composta por cinco componentes críticos: dois elementos chaves da cultura (OPM e empreendedorismo) e três elementos do clima: liderança facilitadora, estrutura orgânica e aberta, e uma abordagem descentralizada do planejamento estratégico. Os autores sugerem ainda a presença de uma sinergia entre a aprendizagem e o desempenho da empresa.

O conceito de aprendizagem organizacional pode ser encontrado no meio acadêmico, definido de formas diversas, complementares e semelhantes como orientação para a aprendizagem (OPA), capacidade de aprendizagem, conhecimento compartilhado, capacidade de aprendizagem organizacional, gestão da aprendizagem entre outros. Nesse estudo são adotados os constructos da OPA (Sinkula et al., 1997; Perin, 2002; Perin, Sampaio, Duha, \& Bittencourt, 2006).

Como forma de desenvolver aspectos estratégicos organizacionais, a OPA é vista como uma postura que visa desenvolver a aprendizagem e a gestão do conhecimento organizacional. A OPA pode ser definida como grupo de valores que influenciam o grau com que uma organização se considera satisfeita com relação às suas teorias em uso (Argyris \& Schon, 1978), seus modelos mentais (De Geus, 1988) e sua lógica dominante (Bettis \& Prahalad, 1995), que pode estar ou não estar baseada no mercado. Organizações com uma forte OPA incentivam, e até mesmo exigem, que seus membros e funcionários constantemente questionem as normas organizacionais vigentes que orientam suas atividades de processamento das informações de mercado, assim como as ações organizacionais (Garvin, 1993; Sinkula, 1994; Sinkula et al., 1997).

Valores que são constantemente associados com as capacidades da aprendizagem organizacional circundam o (a) compromisso com a aprendizagem; (b) mente aberta; e (c) visão compartilhada (Senge, 1990; Sinkula et al., 1997). Empresas que estão comprometidas com a aprendizagem valorizam a necessidade de entender as causas e efeitos de suas ações (Shaw \& Perkins, 1991), já que essa postura é necessária para que as empresas possam detectar e corrigir os erros de suas teorias em uso.

O compromisso com a aprendizagem diz respeito aos princípios da aprendizagem discutidos por Senge (1990). Mente aberta está associada à ideia de desaprender a aprender. O conceito de desaprendizagem (Nystrom \& Starbuck, 1984) pode ser encarado como o centro da mudança organizacional e o processo de aperfeiçoamento e aprendizagem. A visão compartilhada gera uma propensão ao compartilhamento de uma lógica dominante e resultados conjuntamente esperados (Dougherty, 1992). A falta de um foco organizacional compreendido de modo geral e compartilhado por todos gera uma redução na motivação para aprender (Tobin, 1993). Essa visão compartilhada proporciona um senso comum de direção e propósito.

Enquanto comportamentos adotados para uma postura orientada para o mercado são mais rapidamente alterados (Dickson, 1996) e proporcionam um efeito positivo na lucratividade da empresa de um modo mais imediato (Narver \& Slater, 1990), a OPA é mais demorada de ser implementada, já que é o resultado de atitudes, compromissos e processos gerenciais cuidadosamente cultivadas e que são desenvolvidos e acumulados ao longo do tempo e de forma progressiva (Garvin, 1993). 
Uma OPM pode gerar aprendizagem em circuito simples (single-loop learning) adaptativa (Narver \& Slater, 1995). Entretanto considera-se que ela não irá levar a organização a uma aprendizagem em circuito duplo (double-loop learning), geradora de novos conhecimentos, a menos que seja acompanhada de uma forte OPA (Argyris \& Schon, 1978; Senge, 1990). Assim, parte-se do pressuposto de que a OPA, como sendo filosofia estratégica organizacional, proporciona condições de obter melhor desempenho organizacional para as empresas. Alguns estudos importantes no Brasil relacionam a Aprendizagem Organizacional $(\mathrm{AO})$ e a Orientação para aprendizagem (OPA) com o desempenho organizacional de forma positiva (Perin, 2002; Perin et al., 2006). Entretanto ainda existe a necessidade de estudos adicionais que confirmem essa relação.

\section{Metodologia}

Quanto aos procedimentos metodológicos, este estudo se configura como uma pesquisa de levantamento do tipo survey (Babbie, 1999), de caráter descritivo, tendo como participantes executivos e tomadores de decisão de nível estratégico e gerencial de empresas localizadas na região central do Rio Grande do Sul. Os dados analisados foram coletados em um levantamento único.

\section{Elaboração do instrumento de coleta de dados}

Para avaliar as questões referentes aos objetivos deste estudo, que são o nível de OPM, OPA e o Desempenho Organizacional percebido, foi elaborado um instrumento de coleta de dados para a mensuração das variáveis pertinentes definidas inicialmente. Conforme sugerido por Hair, Anderson, Tathan e Black (1998) e Garver e Mentzer (1999), a avaliação e validação do instrumento foi realizada por meio do cálculo do valor de Alfa de Cronbach (Cronbach, 1951), da confiabilidade composta e de variância extraída (AVE), sendo esses dois últimos efetuados a partir da soma das cargas das variâncias padronizadas e dos erros de mensuração das variáveis.

A escala de avaliação adotada para mensurar o nível de OPM foi a MARKOR (Kohli et al., 1993) com os 20 itens sendo avaliados por meio de uma escala Likert de 5 pontos (1=discordo totalmente / $5=$ concordo totalmente). As dimensões da escala são: geração de inteligência (6 itens); disseminação de inteligência (5 itens); e responsividade ( 9 itens). Já a escala de avaliação adotada para mensurar o nível de OPA foi a escala originalmente desenvolvida e adotada por Sinkula et al. (1997). Os 18 itens do instrumento que mensuram a OPA também foram avaliados por meio de uma escala Likert, de concordância de 5 pontos. As dimensões da escala são o Comprometimento para com a Aprendizagem (6 itens); Visão Compartilhada (6 itens); e "Mente Aberta" (6 itens).

Já o desempenho organizacional percebido pelos empresários pesquisados (variável latente dependente) foi mensurado e avaliado por meio de itens selecionados e já utilizados por Kohli et al. (1993) e Narver e Slater (1990): os itens visam mensurar a percepção de lucratividade da organização. Também se utilizaram variáveis adotadas por Baker e Sinkula (1999) para avaliar a situação do segmento de atuação das empresas, para melhor avaliar seus desempenhos. A mensuração de cada um dos itens do constructo do Desempenho Organizacional foi também realizado por meio de escala Likert de 5 pontos ( $1=$ péssimo / $5=$ =xcelente), seguindo recomendações identificadas na revisão da literatura e em outros estudos semelhantes; e respeitando a familiaridade dos respondentes com a escala de cinco pontos.

Tendo como objetivo de caracterizar o perfil das empresas pesquisadas, foram coletadas também informações a respeito da receita anual, do número de funcionários e da quantidade de níveis organizacionais. Estas informações são apresentadas de modo descritivo. 


\section{Coleta de dados}

O survey aplicado na região central do RS contou com a adesão voluntária de 123 respondentes. $\mathrm{O}$ procedimento amostral para este estudo foi uma amostragem não-probabilística por adesão; foram enviados questionários por e-mail para cadastros de empresários obtidos em órgão como AJESM (Associação de Jovens Empreendedores de Santa Maria), CACISM (Câmara da Indústria e Comércio de Santa Maria) e FIERGS (Federação da Indústria do Estado do Rio Grande do Sul). Também foram obtidas respostas por meio de aplicação presencial de questionário, onde foram abordados empresários da região da pesquisa na FEISMA 2010 (evento promovido anualmente pela CACISM).

\section{Análise de dados, método de estimação e medidas de ajustamento}

Inicialmente, os dados de entrada foram submetidos a uma análise de normalidade, onde foram observados os valores de assimetria e curtose. Esta análise apresentou a ocorrência de nãonormalidade em algumas das variáveis. Para fins de análise e adoção do melhor método estatístico de análise, dado o diagnóstico de não-normalidade moderada (assimetria menor ou igual a dois e curtose menor ou igual a sete), o tamanho de amostra inferior a 200 casos e o uso de variáveis categóricas do tipo Likert, com cinco ou mais pontos, foi adotada a abordagem maximum likelihood (ML), considerando a variável categórica como intervalar (West, Finch, \& Curran, 1995).

Na sequência, foram observados e analisados, de forma descritiva, os dados relativos ao perfil das empresas que aderiram à pesquisa. Também foram analisados os valores de média, desvio padrão dos 20 itens da escala MARKOR, dos 18 itens da escala de OPA e dos 4 itens que mensuraram o desempenho organizacional.

No concernente às técnicas de análise multivariada dos dados, realizou-se a análise de componentes principais dos 4 itens que mensuraram o desempenho organizacional, assim como uma redução de itens para as 3 dimensões originais da escala MARKOR (Bagozzi \& Edwards, 1998). Esse procedimento de redução de variáveis também foi adotado nas questões que mensuraram a OPA. Também foi realizada uma análise de regressão múltipla tendo como variáveis independentes as 6 dimensões das escalas de OPM (3 dimensões) e OPA (3 dimensões); e, como variável dependente, a variável originária da redução dos quatro itens de avaliação do desempenho organizacional para um único componente principal.

Finalizando a análise dos dados obtidos, realizou-se uma análise fatorial confirmatória da escala de OPA e OPM, assim como a modelagem de equações estruturais de 3 modelos alternativos de estudo, os quais podem ser visualizados esquematicamente na Figura 1. 


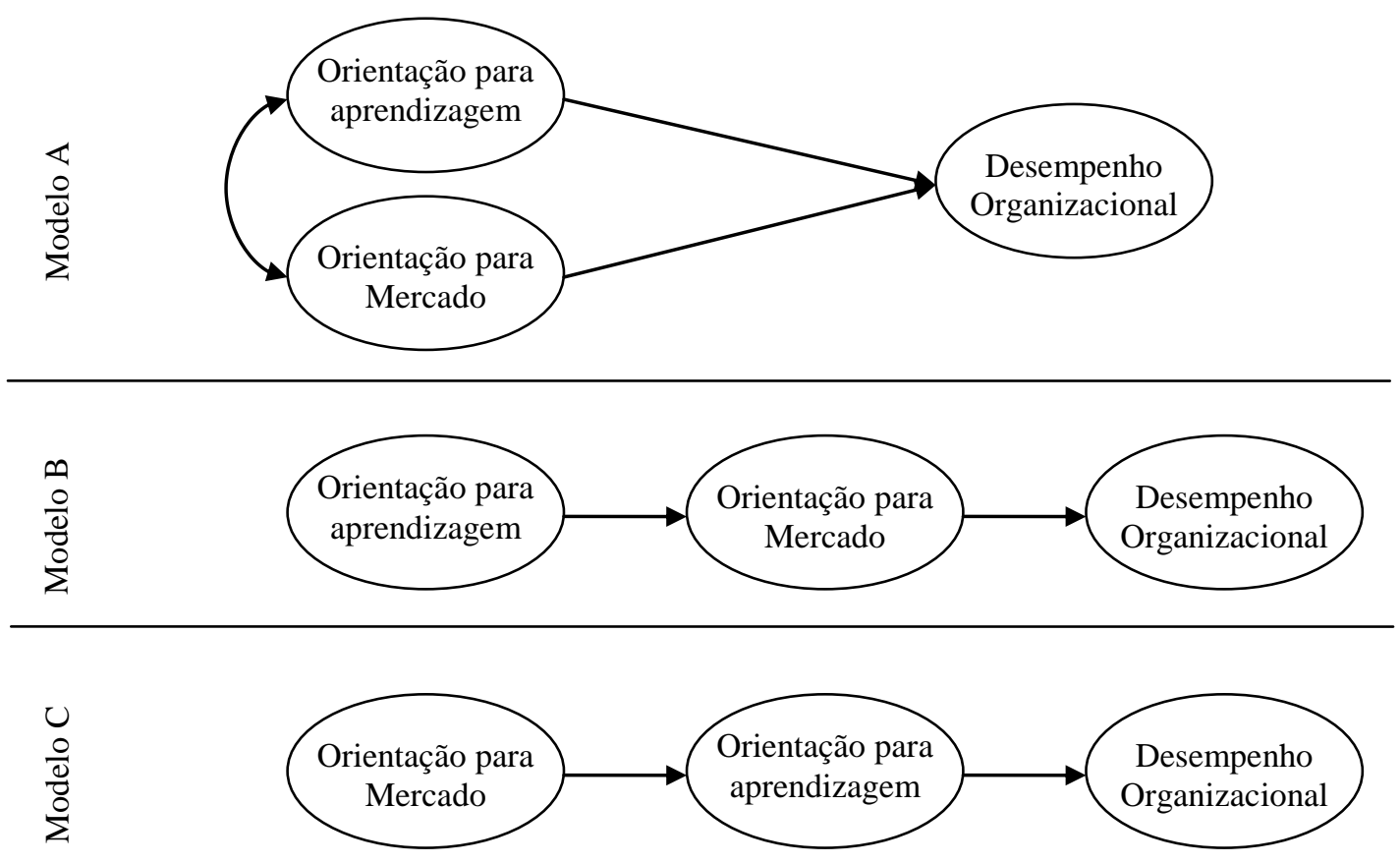

Figura 1. Modelos de Estudo.

O Modelo A se propõe a analisar a relação direta existente entre a OPM e a OPA (Variáveis Independentes) e o Desempenho Organizacional (Variável Dependente). Ainda observa a correlação/covariância existente entre as duas orientações investigadas. O Modelo B observa a influência da OPA no Desempenho Organizacional, tendo como variável interveniente a OPM. E, finalmente, o Modelo C observa a influência da OPM no Desempenho Organizacional, tendo como variável interveniente a OPA.

Para o teste dos modelos analisados foram observados o Qui-quadrado sobre graus de liberdade $\left(\chi^{2} / \mathrm{GL}\right)$, o Goodness-of-fit Index (GFI) e o Root Mean Square Error of Approximation (RMSEA). Já as medidas comparativas de ajustamento, que comparam o modelo proposto ao modelo nulo (null model), foram o Adjusted Goodness-of-fit (AGFI), o Tucker-Lewis Index (TLI) e o Comparative Fit Index (CFI). De acordo com Hair et al. (1998) e Garson (2011), considera-se aceitável o ajustamento de um modelo quando o $\chi^{2} /$ GL apresenta valor igual ou inferior a 5; quando os índices GFI, AGFI, TLI e CFI apresentam valores iguais ou superiores a 0,8 ; e quando o RMSEA apresenta valores entre 0,05 e 0,08 . Os valores foram obtidos por meio de uma Análise Fatorial Confirmatória, e seus resultados são apresentados na próxima seção.

Para fins de análise estatística dos dados coletados, contou-se com o auxílio dos softwares SPSS for Windows 15.0 e o AMOS 7.0.

\section{Resultados do Estudo}

Inicialmente são apresentadas as informações referentes ao perfil das empresas que aderiram ao estudo. Em seguida foram analisados os dados quantitativos obtidos, referentes às variáveis centrais do estudo utilizando.

\section{Caracterização das empresas}

Quanto à receita anual das empresas participantes do estudo que divulgaram suas faixas de receita, ficou constatado que 31,2\% possuem uma receita de até $\mathrm{R} \$ 244$ mil; 33,3\% possuem uma 
receita entre $\mathrm{R} \$ 240$ mil e $\mathrm{R} \$ 1,2$ milhão; $18,3 \%$ estão na faixa de $\mathrm{R} \$ 1,2$ milhão e $\mathrm{R} \$ 10,5$ milhões; 7,5\% têm receita entre $R \$ 10,5$ milhões e $R \$ 60$ milhões; e 9,7\% possuem uma receita acima de $R \$ 60$ milhões. Foi observado também que 30 respondentes não divulgaram essa informação.

Já com relação ao número de funcionários das empresas estudadas, constatou-se que 55,6\% possuem até 19 funcionários; 28,3\% têm entre 20 e 99 funcionários; 10,1\% possuem entre 100 e 499 funcionários; $4 \%$ têm de 500 até 1.000 funcionários; e apenas $2 \%$ contam com mais de 1.000 funcionários. Observou-se também que 24 respondentes não divulgaram essa informação.

Quanto à quantidade de níveis da estrutura organizacional (níveis hierárquicos), constata-se que $76 \%$ das empresas participantes do estudo possuem até 3 níveis: operacional, gerencial e presidência. Outros 19,8\% dos respondentes atuam em empresas com 4 ou 5 níveis organizacionais: operacional, supervisores, gerencial, diretoria, presidência. E apenas 4,2\% dos respondentes representam empresas que possuem 6 níveis ou mais: operacional, supervisores, assistentes, gerencial, diretoria, presidência. Salienta-se que 27 respondentes não responderam à questão referente à quantidade de níveis de suas respectivas organizações.

Portanto, de modo geral, é possível observar que as empresas que aderiram à pesquisa, em sua grande maioria, são empresas de micro, pequeno e médio porte com receitas anuais de até $\mathrm{R} \$ 10,5$ milhões $(82,8 \%)$, contando com até 99 colaboradores $(83,9 \%)$ e possuindo até 3 níveis organizacionais $(76 \%)$.

\section{Análise fatorial e redução de itens dos constructos do estudo}

Os valores de assimetria e curtose observados são adequados para se trabalhar com o método de Maximum Likelihood para a estimação do modelo: assimetria menor do que 2 e curtose menor do que 7. O valor de Alfa de Cronbach da escala MARKOR obtido foi 0,886 e o da escala de OPA foi 0,888, sendo ambos considerados valores altamente positivos, que determinam a confiabilidade interna do instrumento.

Por meio da análise fatorial, os 20 itens da escala original foram reduzidos para 3 novas variáveis, que representam as dimensões da OPM: Geração de Inteligência, Disseminação de Inteligência e Responsividade. Inicialmente foram reduzidos os 6 primeiros itens, que originalmente mensuram a geração de inteligência para uma única variável: Geração de Inteligência. Foram obtidos os valores do Autovalor (Eigenvalue) para a análise fatorial (2,376); o Alfa de Cronbach para a análise da Confiabilidade Interna (0,687); a Porcentagem da Variância Extraída (39,6\%); e a Confiabilidade Composta $(0,793)$. A verificação da adequação da amostra à análise fatorial foi avaliada pelo Teste de esfericidade de Bartlett e pelo índice Kaiser-Meyer-Olkin (KMO), os quais se apresentaram plenamente satisfatórios: $\chi^{2}=142,99, \mathrm{p}<0,001 ; \mathrm{KMO}=0,670$.

Dando sequência à redução de itens, por meio da análise fatorial também foram reduzidos os 5 itens da escala que originalmente mensuram a disseminação de inteligência, para uma variável latente denominada de Disseminação de Inteligência. Foram obtidos os valores do Autovalor (Eigenvalue) para a análise fatorial $(2,529)$, o Alfa de Cronbach para a análise da Confiabilidade Interna $(0,745)$, a Porcentagem da Variância Extraída $(50,58 \%)$ e a Confiabilidade Composta $(0,831)$, seguindo os passos adotados no constructo anterior. O Teste de esfericidade de Bartlett e o índice Kaiser-MeyerOlkin (KMO) apresentaram valores altamente satisfatórios: $\chi^{2}=141,33 ; \mathrm{p}<0,001 ; \mathrm{KMO}=0,764$.

Finalmente, compondo o último constructo da OPM, foram reduzidos os 9 últimos itens da escala, que originalmente mensuram a responsividade, para uma única variável por meio da análise fatorial, a qual foi denominada de Responsividade. Foram obtidos os valores do Autovalor (Eigenvalue) para a análise fatorial $(3,511)$, o Alfa de Cronbach para a análise da Confiabilidade Interna $(0,777)$, a Porcentagem Variância Extraída $(39,02 \%)$ e a Confiabilidade Composta $(0,844)$. O Teste de esfericidade de Bartlett e o índice Kaiser-Meyer-Olkin (KMO) apresentaram valores altamente satisfatórios: $\chi^{2}=260,12 ; \mathrm{p}<0,001 ; \mathrm{KMO}=0,815$. 
Seguindo o mesmo procedimento adotado na redução de itens da escala MARKOR, foram reduzidos os 18 itens da escala de OPA para suas três dimensões originais. Por meio da análise fatorial os 6 itens da escala que originalmente mensuram o comprometimento com a aprendizagem foram reduzidos para uma única variável, denominada de Comprometimento com a Aprendizagem. Observou-se o Eigenvalue para a análise fatorial $(2,934)$, o Alfa de Cronbach para a análise da Confiabilidade Interna $(0,780)$, a Porcentagem da Variância Extraída $(48,90 \%)$ e a Confiabilidade Composta $(0,847)$. Também o Teste de esfericidade de Bartlett e o índice Kaiser-Meyer-Olkin (KMO) apresentaram valores plenamente satisfatórios: $\chi^{2}=227,09 ; \mathrm{p}<0,001 ; \mathrm{KMO}=0,742$.

Por intermédio da análise fatorial também foram reduzidos os 6 itens da escala que originalmente mensuram a visão compartilhada para uma única variável (Visão Compartilhada). Foram verificados o Eigenvalue $(3,435)$, o Alfa de Cronbach para a análise da Confiabilidade Interna $(0,844)$, a porcentagem da Variância Extraída $(57,25 \%)$ e a Confiabilidade Composta $(0,887)$. O Teste de esfericidade de Bartlett e o índice Kaiser-Meyer-Olkin (KMO) apresentaram valores altamente satisfatórios: $\chi^{2}=298,72 ; \mathrm{p}<0,001 ; \mathrm{KMO}=0,791$.

Finalmente, compondo o último constructo da OPA, os 6 últimos itens da escala que originalmente mensuram a postura de mente aberta foram reduzidos para uma única variável por meio da análise fatorial, a qual foi denominada de Mente Aberta. Foram calculados o Eigenvalue para a análise fatorial (2,823), o Alfa de Cronbach para a análise da Confiabilidade Interna $(0,751)$, a Porcentagem da Variância Extraída $(47,05 \%)$ e a Confiabilidade Composta $(0,831)$. O Teste de esfericidade de Bartlett e o índice Kaiser-Meyer-Olkin (KMO) apresentaram valores altamente satisfatórios: $\chi^{2}=205,24 ; \mathrm{p}<0,001 ; \mathrm{KMO}=0,743$.

Finalizando a redução de itens do instrumento de coleta de dados do estudo realizou-se a análise fatorial do constructo utilizado para avaliar o Desempenho Organizacional, percebido pelos respondentes. Por meio da análise fatorial, os 4 itens que avaliam o desempenho foram reduzidos para apenas uma variável latente, denominada de Desempenho Organizacional. São observados o Eigenvalue para a análise fatorial $(2,426)$, o Alfa de Cronbach para a análise da Confiabilidade Interna $(0,765)$, a Porcentagem da Variância Extraída $(60,66 \%)$ e a Confiabilidade Composta $(0,859)$. Também o Teste de esfericidade de Bartlett e o índice KMO foram satisfatórios: $\chi^{2}=150,85$; $<<0,001$; $\mathrm{KMO}=0,716$.

\section{Modelagem de equação estrutural}

Nesta subseção, analisa-se a validade dos Modelos Teóricos A, B e C do Estudo, tendo como Variáveis Independentes (ou intervenientes) a OPM e a OPA; e como Variável Dependente, o Desempenho Organizacional. A análise fatorial confirmatória das relações existentes entre os constructos do estudo assim como os testes de hipóteses para verificar a significância podem ser observados na Tabela 1.

Tabela 1

Estimativas e Testes das Hipóteses da Análise Fatorial Confirmatória dos Constructos do Estudo

\begin{tabular}{llcccc}
\hline & & & Estimativa & Estimativa & Erro \\
& & & Estatística \\
& & & Padronizada & Padrão & Teste \\
\hline DM39 & $\leftarrow$ Desempenho Organizacional & 1,000 & 0,799 & & \\
DM40 & $\leftarrow$ Desempenho Organizacional & 1,071 & 0,794 & 0,137 & $7,830 *$ \\
DM41 & $\leftarrow$ Desempenho Organizacional & 0,803 & 0,710 & 0,111 & $7,257 *$ \\
DM42 & $\leftarrow$ Desempenho Organizacional & 0,661 & 0,441 & 0,148 & $4,462 *$ \\
\hline
\end{tabular}


Tabela 1 (continuação)

\begin{tabular}{|c|c|c|c|c|c|c|}
\hline & & & Estimativa & $\begin{array}{l}\text { Estimativa } \\
\text { Padronizada }\end{array}$ & $\begin{array}{c}\text { Erro } \\
\text { Padrão }\end{array}$ & $\begin{array}{c}\text { Estatística } \\
\text { Teste }\end{array}$ \\
\hline Geração de Inteligência & $\leftarrow$ & OPM & 1,000 & 0,801 & & \\
\hline $\begin{array}{l}\text { Disseminação de } \\
\text { Inteligência }\end{array}$ & $\leftarrow$ & OPM & 1,065 & 0,845 & 0,109 & $9,791 *$ \\
\hline Responsividade & $\leftarrow$ & OPM & 1,060 & 0,845 & 0,108 & $9,771 *$ \\
\hline Mente Aberta & & OPA & 1,000 & 0,713 & & \\
\hline Visão Compartilhada & $\leftarrow$ & OPA & 1,161 & 0,839 & 0,163 & $7,141 *$ \\
\hline $\begin{array}{l}\text { Comprometimento com } \\
\text { a Aprendizagem }\end{array}$ & & OPA & 0,770 & 0,551 & 0,146 & $5,258 *$ \\
\hline
\end{tabular}

* Significativo ao nível de $\mathrm{p}<0,001$.

Pode ser observado que, por meio dos testes de hipóteses realizados na Análise Fatorial Confirmatória da escala MARKOR, da escala de OPA e do constructo de Desempenho Organizacional, todas as variáveis latentes são significativas em nível de $p<0,001$. Assim, os itens são válidos (variáveis latentes) para mensurar os constructos da OPM, da OPA e do Desempenho Organizacional.

Já com relação aos Modelos de Estudo, algumas diferenças significativas são observadas. Os resultados das relações propostas nos três modelos apresentados anteriormente são apresentados na Tabela 2.

Tabela 2

Estimativas e Testes das Hipóteses das Ligações entre Construtos dos Três Modelos Testados

\begin{tabular}{|c|c|c|c|c|c|c|c|}
\hline MODELO & & & & Estimativa & $\begin{array}{l}\text { Estimativa } \\
\text { Padronizada }\end{array}$ & $\begin{array}{c}\text { Erro } \\
\text { Padrão }\end{array}$ & $\begin{array}{c}\text { Estatística } \\
\text { Teste }\end{array}$ \\
\hline \multirow{3}{*}{ MODELO A } & OPA & $\leftarrow$ & OPM & $0,456(\mathrm{cov})$ & $0,803(\rho)$ & 0,092 & $4,937 *$ \\
\hline & Desempenho Organizacional & $\leftarrow$ & OPA & 0,064 & 0,089 & 0,165 & 0,389 \\
\hline & Desempenho Organizacional & $\leftarrow$ & OPM & 0,236 & 0,366 & 0,145 & 1,634 \\
\hline \multirow{2}{*}{ MODELO B } & OPM & $\leftarrow$ & OPA & 0,904 & 0,806 & 0,144 & $6,268 *$ \\
\hline & Desempenho Organizacional & $\leftarrow$ & OPM & 0,285 & 0,443 & 0,070 & $4,073 *$ \\
\hline \multirow{2}{*}{ MODELO C } & OPA & $\leftarrow$ & OPM & 0,736 & 0,818 & 0,113 & $6,483 *$ \\
\hline & Desempenho Organizacional & $\leftarrow$ & OPA & 0,315 & 0,433 & 0,085 & $3,721 *$ \\
\hline
\end{tabular}

* Significativo ao nível de $\mathrm{p}<0,001$.

No Modelo A, nenhuma das variáveis independentes têm impacto significativamente positivo no Desempenho das organizações estudadas. Entretanto observaram-se os valores de covariância $(\operatorname{cov}=0,456)$ e correlação $(\rho=0,803)$ existentes entre os constructos como sendo significativos no nível de $\mathrm{p}<0,01$. Assim, o Modelo A não apresenta resultados significativos positivos, podendo ser considerado modelo estatisticamente frágil.

Já no Modelo B, a OPA exerce uma influência positiva e significativa $(\mathrm{p}<0,001)$ na OPM. Esta, por sua vez, exerce uma influência também positiva e significativa $(\mathrm{p}<0,001)$ no Desempenho Organizacional. Dessa forma, o Modelo B apresenta resultados significativos positivos; pode ser considerado modelo estatisticamente mais consolidado. É sugerido então que a OPA, intermediada 
pela OPM, exerce influência positiva no desempenho organizacional das empresas pesquisadas. $\mathrm{O}$ Modelo B também conta com um suporte teórico de autores que afirmam que a OPA pode ser considerada como orientação mais intrínseca da organização, devendo ser encarada como filosofia ou postura enraizada na cultura organizacional. Já a OPM é encarada como conjunto de posturas e ações pragmáticas de gestão, que conferem a seus adotantes possíveis desempenhos superiores perante a concorrência. Portanto, parece lógico supor que a OPA seja intermediada pela OPA.

Finalmente, no Modelo C, foi constatado que a OPM exerce influência positiva e significativa $(\mathrm{p}<0,001)$ na OPA. Essa última, por sua vez, exerce influência também positiva e significativa $(\mathrm{p}<0,001)$ no Desempenho Organizacional. Os resultados do Modelo reforçam os resultados do estudo de Jiménez e Navarro (2007), que sugere que a OPM influencia significativamente o desempenho organizacional apenas quando intermediado pela OPA. Assim, o Modelo C apresenta resultados significativos positivos; pode ser considerado um modelo estatisticamente consolidado, a ser confrontado com o Modelo B.

Na Figura 2 são apresentados os resultados obtidos para o teste dos três modelos já apresentados na tabela anterior; considera-se que o formato gráfico facilita a visualização do relacionamento entre as variáveis do estudo.
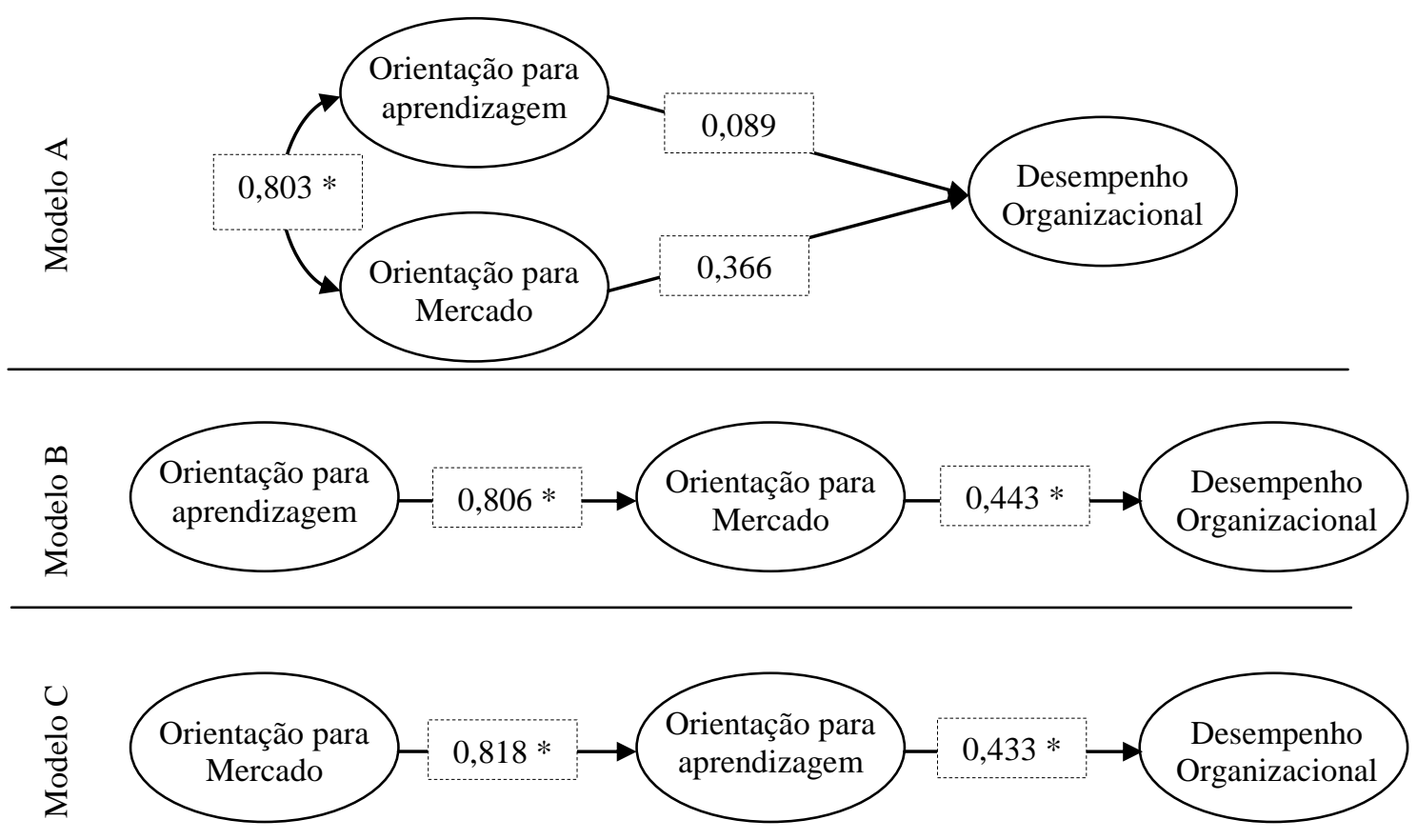

Figura 2. Resultado dos Modelos de Estudo com Estimativas Padronizadas.

* Significativo ao nível de $\mathrm{p}<0,001$.

Em termos conceituais e teóricos, o Modelo B é embasado por pressupostos teóricos já apresentados; é considerado inicialmente como o modelo de maior validade estatística e conceitual. Entretanto, a confirmação de sua validade, em comparação com os demais modelos testados, é feita por meio da observação dos índices de ajustamento apresentados na Tabela 3 a seguir. 
Tabela 3

Índices de Ajustamento dos Modelos de Estudo

\begin{tabular}{lccc}
\hline & Modelo A & Modelo B & Modelo C \\
\hline $\mathbf{X}^{2} /$ GL & 2,079 & $\mathbf{2 , 0 2 0}$ & 2,092 \\
GFI & 0,877 & $\mathbf{0 , 8 7 7}$ & 0,872 \\
AGFI & 0,789 & $\mathbf{0 , 7 9 5}$ & 0,787 \\
TLI & 0,878 & $\mathbf{0 , 8 8 5}$ & 0,876 \\
CFI & 0,929 & $\mathbf{0 , 9 3 1}$ & 0,926 \\
RMSEA & 0,094 & $\mathbf{0 , 0 9 1}$ & 0,095 \\
\hline
\end{tabular}

Pode ser observado que os índices de ajustamento do Modelo B de Estudo não se apresentam como plenamente satisfatórios, de acordo com os pressupostos defendidos por Hair et al. (1998) e Garson (2011). Entretanto, são os valores considerados mais adequados, quando se comparam os índices de ajustamento dos três modelos testados. O valor de $\mathrm{X}^{2} / \mathrm{GL}$ está de acordo com os pressupostos dos autores assim como o CFI. Os demais valores optidos para o GFI, AGFI e TLI estão um pouco abaixo do definido como aceitável, assim como o RMSEA está acima de 0,08 (erro do modelo). Todavia os valores do GFI, AGFI, TLI e CFI no Modelo B são ligeiramente mais elevados, e o valor do RMSEA é ligeiramente inferior aos demais. Assim, entre os modelos testados, o Modelo B é considerado o mais adequado. Contudo, devido ao não alcance da total satisfatoriedade dos índices de ajustamento, os modelos podem ser considerados como limitados; mas, de certa forma, sua validade merece reconhecimento.

\section{Conclusões e Discussões}

Devido à alta competitividade instaurada entre as organizações de nível global e as crescentes exigências do mercado por produtos e serviços de maior qualidade, as organizações precisam implementar estratégias inovadores e adaptativas. $\mathrm{O}$ foco nas necessidades do mercado, assim como no monitoramento constante da concorrência são ações elementares para a obtenção do sucesso empresarial e alta performance organizacional. Dentre as estratégias e orientações empresariais, com foco no aumento das condições competitivas, têm-se a OPM e a OPA. Ambas as orientações são objetos de estudos constantes no meio acadêmico e têm despertado interesse de pesquisadores, que buscam consolidar e sedimentar o conhecimento construído sobre essa temática.

Entretanto, observam-se ainda certas lacunas nesse campo de estudos, que ainda carece de pesquisas adicionais. Estudos regionais e focados em setores específicos ainda são necessários. Assim, o objetivo geral deste estudo foi o de identificar de que forma a OPA e a OPM se relacionam entre si e influenciam o desempenho das empresas da região central do Rio Grande do Sul. A investigação de determinadas práticas estratégicas organizacionais proporciona melhores condições de entendimento das relações existentes entre essas práticas e a alta performance organizacional objetivada pelas empresas.

A partir dessa investigação, verificou-se que a OPM exerce uma influência positiva e significativa no desempenho organizacional das empresas pesquisadas, as quais são, em sua grande maioria, Micro, Pequenas e Médias Empresas (MPMEs). Em contrapartida, a OPA não apresentou resultados semelhantes, não demonstrando impacto significativo no desempenho organizacional. Subdividindo as orientações investigadas nas dimensões que as compõem (variáveis latentes), e efetuando-se uma análise de regressão linear múltipla, observou-se que, na OPM, nenhuma das dimensões, de forma isolada, exerce impacto significativo no desempenho organizacional. Já dentre as 
3 dimensões da OPA, observou-se que o comprometimento com a aprendizagem e a "mente aberta" apresentam impacto positivo e significativo no desempenho das organizações pesquisadas. Apesar de os índices de ajustamento observados na MEE não terem sido plenamente satisfatórios, o estudo apresenta resultados relevantes, que confirmam algumas premissas e reforçam alguns resultados obtidos em outros estudos acadêmicos.

Ao testar os três modelos propostos no estudo, observou-se que a maior significância existe quando uma orientação intermedeia a outra. Assim, a OPM influencia significativamente o Desempenho Organizacional, quando intermediada pela OPA. Da mesma forma, a OPA só exerce influência significativa no desempenho das organizações pesquisadas, quando intermediada pela OPM. O modelo que apresentou os melhores resultados e índices de ajustamento foi o Modelo B, que trata a OPA como variável independente, e a OPM como variável interveniente.

A contribuição prática deste estudo é proporcionar uma visão estratégica aos executivos e gestores organizacionais de que as orientações estratégicas, com foco no mercado e na aprendizagem, proporcionam impactos positivos significativos no desempenho dos negócios. Entretanto, esses impactos são potencialmente maiores, quando as orientações para o mercado e para a aprendizagem são adotadas de forma sinérgica e interdependente. Logo, a interdependência, observada entre as orientações investigadas, demonstra oportunidades de alavancagens estratégicas por meio de práticas que fomentem a gestão do conhecimento organizacional e de mercado.

Como limitação importante deste estudo, é salientada a amostra por adesão de empresas de portes e segmentos variados, o que prejudica a capacidade de generalização dos resultados do estudo, devido à diversidade de empresas e práticas organizacionais heterogêneas. Sugerem-se estudos futuros sobre a temática em questão, sendo consideradas outras variáveis e orientações que possam afetar de forma significativa o modelo; como, por exemplo, inovação, perfil empreendedor, práticas de desenvolvimento sustentável e orientação para o relacionamento. Também se sugerem estudos que investiguem empresas de um mesmo setor, assim como a investigação dos impactos dessas orientações, ao longo de cadeias produtivas e redes de organização.

\section{Artigo recebido em 31.05.2011. Aprovado em 09.11.2011.}

\section{Referências}

Antoni, V. L. (2004). A relação entre orientação para o mercado e performance organizacional: um estudo nos cursos de bacharelado em Administração da região Sul do Brasil (Tese de doutorado). Universidade Federal de Santa Catarina, Florianópolis, SC, Brasil.

Argyris, C., \& Schon, D. A. (1978). Organizational learning: a theory of action perspective. Reading, MA: Addison-Wesley.

Armario, J. M., Ruiz, D. M., \& Armario, E. M. (2008). Market orientation and internationalization in small and medium-sized enterprises. Journal of Small Business Management, 46(4), 485-511. doi: 10.1111/j.1540-627X.2008.00253.x

Babbie, E. (1999). Métodos de pesquisa de survey. Belo Horizonte: Ed. UFMG.

Bagozzi, R. P., \& Edwards, J. R. (1998). A general approach for representing constructs in organizational research. Organizational Research Methods, 1(1), 45-87. doi: $10.1177 / 109442819800100104$

Baker, W. E., \& Sinkula, J. M. (1999). The synergetic effect of market orientation and learning orientation on organizational performance. Journal of the Academy of Marketing Science, 27(4), 411-427. doi: 10.1177/0092070399274002 
Bettis, R. A., \& Prahalad, C. K. (1995). The dominant logic: retrospective and extension. Strategic Management Journal, 16(1), 5-14. doi: 10.1002/smj.4250160104

Cantalone, R. J., Cavusgil, S. T., \& Zhao, Y. (2002). Learning orientation, firm innovation capability, and firm performance. Industrial Marketing Management, 31(6), 515-524. doi: 10.1016/S00198501(01)00203-6

Cronbach, L. J. (1951). Coefficient alpha and the internal structure of tests. Psychometrika, 16(3), 297-334. doi: 10.1007/BF02310555

Day, G. S. (2001). A empresa orientada para o mercado: compreender, atrair e manter clientes valiosos. Porto Alegre: Bookman.

De Geus, A. P. (1988). Planning as learning? Harvard Business Review, 66(2), 70-74.

Dickson, P. R. (1996). The static and dynamic mechanics of competition: a comment on hunt and Morgan's comparative advantage theory. Journal of Marketing, 60(4), 102-106. doi: $10.2307 / 1251904$

Dougherty, D. (1992). Interpretive barriers to successful product innovation in large firms. Organization Science, 3(2), 179-202. doi: 10.1287/orsc.3.2.179

Eboli, M. (2004). Educação corporativa no Brasil: mitos e verdades. São Paulo: Editora Gente.

Ellis, P. D. (2006). Market orientation and performance: a meta-analysis and cross-national comparisons. Journal of Management Studies, 43(5), 1089-1107. doi: 10.1111/j.14676486.2006.00630.x

Fleury, M. T., \& Oliveira, M., Jr. (2001). Gestão estratégica do conhecimento. São Paulo: Editora Atlas.

Garson, G. D. (2011). Structural equation modeling. Recuperado em 15 janeiro, 2011, de http://www2.chass.ncsu.edu/garson/pa765/structur.htm

Garver, M. S., \& Mentzer, J. T. (1999). Logistics research methods: employing structural equation modeling to test for construct validity. Journal of Business Logistics, 20(1), 33-57.

Garvin, D. A. (1993). Building a learning organization. Harvard Business Review, 71(4), 78-91.

Hair, J. F., Anderson, R. E., Tathan, R. L., \& Black, W. C. (1998). Multivariate data analysis. New Jersey: Prentice Hall.

Jaworski, B. J., Macinnis, D. J., \& Kohli, A. K. (2002). Generating competitive intelligence in organizations. Journal of Market-Focused Management, 5(4), 279-307. doi: 10.1023/B:JMFM.0000008071.19917.36

Jiménez, D. J., \& Navarro, J. G. C. (2007). The performance effect of organizational learning and market orientation. Industrial Marketing Management, 36(6), 694-708. doi: 10.1016/j.indmarman.2006.02.008

Khandekar, A., \& Sharma, A. (2006). Organizational learning and performance: understanding the Indian scenario in present global context. Education \& Training, 48(8), 682-692. doi: $10.1108 / 00400910610710092$

Kohli, A. K., \& Jaworski, B. J. (1990). Market orientation: the construct, research propositions, and managerial implications. Journal of Marketing, 54(2), 1-18. doi: 10.2307/1251866

Kohli, A. K., Jaworski, B. J., \& Kumar, A. (1993). Markor: a measure of market orientation. Journal of Marketing Research, 30(4), 467-477. doi: 10.2307/3172691 
Kolb, D. A. (1984). Experiential learning: experience as the source of learning and development. New Jersey: Prentice Hall.

Leopoldino, C. B., \& Loiola, E. (2010, setembro). Desempenho organizacional e aprendizagem organizacional: o que podemos aprender sobre essa relação? Anais do Encontro Nacional da Associação Nacional de Pós-Graduação e Pesquisa em Administração, Rio de Janeiro, RJ, Brasil, 34.

Liao, S. H., Chang, W. J., Wu, C. C., \& Katrichis, J. M. (2011). A survey of market orientation research (1995-2008). Industrial Marketing Management, 40(2), 301-310. doi: 10.1016/j.indmarman.2010.09.003

Mandelli, M. (1999). Influência da orientação para o mercado sobre o crescimento de vendas nas maiores empresas privadas indústrias metalúrgicas, mecânicas e de material elétrico de Caxias do Sul (Dissertação de mestrado). Universidade Federal do Rio Grande do Sul, Porto Alegre, RS, Brasil.

Menna, H. L. (2001, setembro). Orientação para mercado e performance: evidências em empresas gaúchas de varejo de confecções masculinas. Anais do Encontro Nacional da Associação Nacional de Pós-Graduação e Pesquisa em Administração, Campinas, SP, Brasil, 25.

Narver, J. C., \& Slater, S. F. (1990). The effect of a market orientation on business profitability. Journal of Marketing, 54(4), 20-35 doi: 10.2307/1251757

Narver, J. C., \& Slater, S. F. (1995). Market orientation and the learning organization. Journal of Marketing, 59(3), 63-74. doi: 10.1108/09696479610106754

Narver, J. C., Slater, S. F., \& Tietje, B. (1998). Creating a market orientation. Journal of MarketFocused Management, 2(3), 241-255. doi: 10.1023/A:1009703717144

Nystrom, P. C., \& Starbuck, W. (1984). To avoid organizational crises, unlearn. Organizational Dynamics, 12(4), 53-65. doi: 10.1016/0090-2616(84)90011-1

Olavarrieta, S., \& Friedmann, R. (2008). Market orientation, knowledge-related resources and firm performance. Journal of Business Research, 61(6), 623-630. doi: 10.1016/j.jbusres.2007.06.037

Perin, M. G. (2002). A relação entre orientação para o mercado, aprendizagem organizacional e performance (Tese de doutorado). Universidade Federal do Rio Grande do Sul, Porto Alegre, RS, Brasil.

Perin, M. G., \& Sampaio, C. H. (2001, setembro). A relação entre as dimensões de orientação para mercado e a performance. Anais do Encontro Nacional da Associação Nacional de PósGraduação e Pesquisa em Administração, Campinas, SP, Brasil, 25.

Perin, M. G., Sampaio, C. H., Duhá, A. H., \& Bittencourt, C. C. (2006). Processo de aprendizagem organizacional e desempenho empresarial: o caso da indústria eletroeletrônica no Brasil. $R A E$ Eletrônica, 5(2). Recuperado de http://www.scielo.br/scielo.php?script=sci_arttext\&pid=S167656482006000200005. doi: 10.1590/S1676-56482006000200005

Perin, M. G., Sampaio, C. H., \& Faleiro, S. N. (2004). O impacto da orientação para o mercado e da orientação para aprendizagem sobre a inovação de produto: uma comparação entre a indústria eletroeletrônica e o setor de ensino universitário de administração. Revista de Administração Contemporânea, 8(1), 79-103. doi: 10.1590/S1415-65552004000100005

Prieto, I. M., \& Revilla, E. (2006). Assessing the impact of learning capability on business performance: empirical evidence from Spain. Management Learning, 37(4), 499-522. doi: $10.1177 / 1350507606070222$ 
Rhee, J., Park, T., \& Lee, D. H. (2010). Drivers of innovativeness and performance for innovative SMEs in South Korea: mediation of learning orientation. Technovation, 30(1), 65-75. doi: 10.1016/j.technovation.2009.04.008

Sampaio, C. H. (2000). Relação entre orientação para o mercado e performance empresarial em empresas de varejo de vestuário do Brasil (Tese de doutorado). Universidade do Rio Grande do Sul, Porto Alegre, RS, Brasil.

Senge, P. M. A. (1990). Quinta disciplina: arte, teoria e prática da organização de aprendizagem. São Paulo: Best Seller.

Shaw, R. B., \& Perkins, D. N. T. (1991). Teaching organizations to learn. Organization Development Journal, 9(4), 1-12.

Silveira, T. (1998, setembro). Verificação do grau de orientação para o mercado em empresas calçadistas do Vale do Rio dos Sinos. Anais do Encontro Nacional da Associação Nacional de Pós-Graduação e Pesquisa em Administração, Foz do Iguaçu, PR, Brasil, 22.

Simon, H. A. (1991). Bounded rationality and organizational learning. Organization Science, 2(1), 125-134. doi: 10.1287/orsc.2.1.125

Sinkula, J. M. (1994). Market information processing and organizational learning. Journal of Marketing, 58(1), 35-45. doi: 10.2307/1252249

Sinkula, J. M., Baker, W., \& Noordewier, T. G. A. (1997). Framework for market-based organizational learning: linking values, knowledge and behavior. Journal of the Academy of Marketing Science, 25(4), 305-318. doi: 10.1177/0092070397254003

Tobin, D. R. (1993). Re-educating the corporation: foundations for the learning organization. Essex Junction, VT: Oliver Wright.

Wang, C. L. (2008). Entrepreneurial orientation, learning orientation, and firm performance. Entrepreneurship Theory and Practice, 32(4), 635-656. doi: 10.1111/j.1540-6520.2008.00246.x

West, S. G., Finch, J. F., \& Curran, P. J. (1995). Structural equation models with nonnormal variables: problems and remedies. In R. H. Hoyle (Ed.), Structural equation modeling: concepts, issues, and applications (pp. 56-75). London: Sage Publications. 